\title{
Chemotherapeutic Trials and their Assessments
}

\author{
R. J. W. REES \\ Laboratory for Leprosy and Mycobacterial Research \\ National Institute for Medical Research \\ Mill Hill, London NW7 1 AA
}

\begin{abstract}
The well-established methods for the conduct and assessment of chemotherapeutic trials in leprosy have more recently been enhanced by the inclusion of the mouse footpad infection. Examples are provided of the use of this infection as a more sensitive method for the assessment of new drugs, their speed of action and the detection of persister viable organisms and the emergence of drug-resistant bacilli. The importance of these results in relation to the value of short-term trials in the initial assessment of a new antileprosy drug and the necessity of very-long-term trials in the final assessment of a new drug or new drug regimen in the treatment of lepromatous leprosy are discussed.
\end{abstract}

In considering chemotherapeutic trials and their assessment in leprosy I will begin by quoting from a recent Editorial in the Lancet (1974): "The treatment of pulmonary tuberculosis is soundly based on the results of controlled clinical trials. Unfortunately it is not always effective, but if it fails the fault lies with the physician, the patient or the medical services. Failure is not due to inefficacy of the drugs or deficiencies of research. With other forms of tuberculosis the position is different. Few control trials have been done." This editorial incidentally referred to tuberculosis of the spine. However, my reason for quoting from this editorial is to compare and contrast the current situation in the chemotherapy of tuberculosis and leprosy. Historically dapsone was shown to be efficacious in the treatment of leprosy long before any antituberculosis treatment, streptomycin, was discovered and yet well defined and precise chemotherapeutic trial methods were evolved for tuberculosis long before they were applied to leprosy. On the other hand, because both infections were caused by a mycobacterium and both were chronic type infections, the well-defined methods for assessing trials in the treatment of leprosy followed the basic principles applied to chemotherapeutic trials in tuberculosis (Doull, 1960; Waters et al 1967). While all would admit in retrospect the need for incorporating well-defined criteria and basic methodology into trials concerned with the chemotherapy of leprosy and the acceptance that both infections were caused by a mycobacterium, the efficacy of therapy then available was entirely different. While it would be true to say that treatiment of leprosy (by dapsone) was not always effective, unlike the chemotherapeutic agents for pulmonary tuberculosis, failure of dapsone therapy in leprosy could 
not be said to be due to the fault of the physician, the patient or the medical services. Clearly, the difference could have arisen from deficiencies in dapsone, compared with antituberculosis drugs, or differences in the capacity of leprosy and tuberculosis patients to respond to chemotherapy.

Both differences are relevant and as such are fundamental to the design of chemotherapeutic trials in leprosy. Considering first the efficacy of dapsone against Mycobacterium leprae, current studies in the mouse indicate that it is a bacteriostatic drug, whereas the major antituberculosis drugs (streptomycin, isoniazid and rifampicin) are bactericidal drugs against Mycobacterium tuberculosis. On the basis of this important difference the antituberculosis drug would be expected to be more effective than would dapsone therapy for leprosy.

The second consideration is concerned with possible differences between leprosy and tuberculosis in the type of infection and the capacity of the patient to respond to chemotherapy. Here there are undoubtedly very great differences, not only between tuberculosis and leprosy but also among patients with leprosy. The appreciation of these differences are fundamental to the selection and basis of the conduct of chemotherapeutic trials in leprosy and the limit to which the methodology for chemotherapeutic trials in tuberculosis can be applied to leprosy. These differences are particularly pertinent when chemotherapeutic trial methods for pulmonary tuberculosis are directly applied to leprosy. Unfortunately, these fundamental differences have not always been appreciated by tuberculosis workers. The basic difference is that while acute pulmonary tuberculosis is a progressive and highly bacilliferous infection, leprosy can present a wide spectrum of disease within which only those patients with the lepromatous type of infection are highly bacilliferous and uniformly progressive. It is now well recognized that the majority of patients with leprosy have less acute infections, with fewer bacilli and where the clinical manifestations predominantly arise from a spontaneous capacity of the host to destroy bacilli. This capacity is greatly enhanced by chemotherapy, whereas the lepromatous patients are almost completely deficient of this capacity, even with chemotherapy. Therefore, in chemotherapeutic trials in leprosy only patients with lepromatous leprosy can be used to be comparable with trials in active pulmonary tuberculosis, although the immunological capacities of the patients will be greater in tuberculosis than in leprosy.

I have chosen to introduce the subject of chemotherapeutic trials and their assessments in leprosy as compared with such trials in tuberculosis because scientific methods for trials in leprosy came from experience gained in tuberculosis. However, once these general principles were applied, which undoubtedly were beneficial, it soon became apparent that there were major differences between the two infections and the type of chemotherapeutic drugs available, which would not be beneficial if strictly applied to leprosy.

In my paper I have followed the guidelines of Professor Freerksen in his opening remarks by assuming that the members of this Colloquium are fully conversant with the leprosy literature, and therefore this is not a review. I shall begin by underlining the general principles to be applied to chemotherapeutic trials and their assessment in lepromatous leprosy, pinpoint these features of leprosy and the drugs available compared with tuberculosis. I shall then present the broad results obtained from shorter and long-term chemotherapeutic trials in leprosy, particularly stressing the application and significance of the footpad infection in mice as more recently applied to these various trials. I will stress the 
difficulties inherent in attempting to assess any new drugs, or drug regimens, as compared with dapsone for "curing" patients with lepromatous leprosy or assessing the emergence of drug resistance.

\section{Standard Requirements for Leprosy Drug Trials}

The requirements for trials in general and for leprosy in particular as proposed respectively by Doull (1960) and Waters et al. (1967), and which have withstood the test of time are summarized in Table 1 . While all these requirements are

TABLE 1

Standard requirements for leprosy drug trials
A. Cases selected must be:
(1) Lepromatous (LL/LI) on Ridley-Jopling scale.
(2) Untreated, with a Morphological Index (MI) of 5 or more.
B. In control trials allocation to treatment groups must be randomized.
C. Evaluation of treatment must be based on independent clinical, bacteriological and histological assessments.

essential to produce reliable and reproducible results, to avoid bias in the assessments and provide comparability between different Centres, the correct selection of lepromatous patients is of overriding importance. There are two reasons for selecting only lepromatous patients, the first is basic to chemotherapy and the second relates to the very variable responses to therapy by nonlepromatous patients. Thus, by definition a chemotherapeutic trial is an assessment of an antibacterial agent, and within the spectrum of leprosy only lepromatous patients have an adequate and inevitably active and progressing bacteriological population on which to assess antibacterial drugs. Tuberculoid patients are excluded because they have too few bacteria and although patients with borderline (BB) or near-lepromatous (BL) leprosy may have relatively high bacterial populations, they are variable and they are killed and eliminated more rapidly and more variably with therapy than they are in lepromatous patients. All these bacteriological variabilities reflect variabilities in the capacity of the host against Myco. leprae and are not a measure of the efficacy of the antibacterial drug per se. It is for these two reasons that only lepromatous patients must be selected for trials concerned with assessing and comparing new antileprosy drugs and comparing them with dapsone. Now that it has been established that the spectrum of leprosy is essentially immunologically determined, the classification and selection of lepromatous patients (LL/LI) for chemotherapeutic trials should be based on the only currently reliable classification which takes into consideration immuno-pathological features (Ridley and Jopling, 1966; Ridley and Waters, 1969).

I have stressed these points, for although they are the basis of current concepts on the pathogenesis of leprosy, they are, unfortunately, still frequently ignored in chemotherapeutic studies.

Of the various assessments carried out in standard chemotherapeutic trials I will only comment on the bacteriological assessments because these are the only ones 
directly concerned with antibacterial activity. Moreover, because Myco. leprae cannot be cultured in vitro indirect methods of assessing viability have had to be developed in place of routine bacteriological cultures, which are applied in chemotherapeutic trials concerned with all other bacterial infections. Thus the Morphological Index (MI) is the only substitute for indirectly determining viability of Myco. leprae in the routinely available skin scrape samples, which are also used for semi-quantitative assessments of the bacteriological load in the skin (Bacteriological Index, BI). Regarding the MI, based routinely on the morphology of 100 acid-fast bacilli, it is only of value for assessing and comparing the rate at which Myco. leprae are killed in the skin in the short initial period. This is because on standard dapsone therapy the MI falls to 0 by 6 months. Since trials must now be concerned with new drugs or drug combinations which are more rapidly killing than dapsone, the MI will be of no value after the first 6 months. At the same time it is essential to appreciate that a MI of 0 at 6 months, based on the assessment of only 100 acid-fast bacilli as against a possible total bacillary population in the patient of $10^{11}$ acid-fast bacilli, does not mean that there are no viable bacilli left-it in fact could mean that there are not more than $10^{9}$ viable bacilli left. On the other hand, assessment of the BI can be continued for many years and in general the rate at which it diminishes, and if it continues to diminish at a steady rate of $1 \mathrm{log}$ per annum, is good evidence that the drug under test is reducing the proportion of living $M y c o$. leprae at a rate comparable to that obtained by dapsone. However, like the MI the BI has a finite value, and using the Ridley scale of $0-6$, which is a logarithmic scale, then a BI of 0 represents less than 1000-100 acid-fast bacilli per gram of skin. Although the MI, as explained, is only a limited measure of viable organisms, and normally has no value after the first 6 months, it must still be assessed, since the reappearance of a positive MI and a rising MI, would indicate relapse, and as long as it could be certain that therapy had been taken, it would indicate the emergence of drug resistance.

Assessments of MI and BI can also be made on histological sections, and methods for these are well defined. Depending on the time spent on examining sections, these assessments can be made somewhat more sensitive than MIs and BIs based on smears; particularly the MI, since the assessment can be made on particular histological sites. This applies to dermal nerves and arrector pili muscle, sites in which morphologically intact bacilli are known to remain for long periods. A few morphologically intact bacilli in these sites are infinitely more sensitive than the examination of a skin scrape where the same bacilli would be overwhelmingly outnumbered by bacilli from the rest of the skin.

Although there is still no in vitro cultivation of Myco. leprae, since 1960 the mouse footpad infection has been available and more recently this technique has been applied to chemotherapeutic trials as a much more sensitive and direct method of assessing viable Myco. leprae than the MI, and the nearest to a routine culture. It is approximately 100 to 1000 times more sensitive than the MI. Moreover, the mouse footpad technique can not only be used to determine viable organisms from human tissues but also to determine their drug sensitivity. It is the application of the mouse footpad technique I will particularly draw upon in the rest of my paper, which will be concerned with short and longer termed chemotherapeutic trials, trials related to the problem of drug resistance and finally speculations on whether patients with lepromatous leprosy can be cured by chemotherapy alone and the need for trials on patients with non-lepromatous leprosy. 


\section{Short-term Pilot Trials}

This type of trial was introduced in 1967 (Waters et al.) as the first type of trial to be used when testing a new antileprosy drug in man. Although the standard requirements were the same as those set out in Table 1, the key assessment is the MI and as this reaches 0 in 4.5-6months with dapsone treatment, this was the period chosen for pilot trials to assess the relative efficacy of new drugs or drug combinations. All drugs used in the pilot trials must have first satisfied the pharmacological and drug safety regulations. The introduction of the pilot type trial preceded the use of the mouse footpad infection as a routine test for screening new antileprosy drugs. Therefore, at that time leprosy in man was being used to identify specific antileprosy activity. This is no longer justified. Now only new drugs that have been fully screened against Myco. leprae in the mouse footpad test, and shown to have activity comparable to dapsone, should be submitted to a pilot trial in man (see Committee on Experimental Chemotherapy, 1974).

Finally, and most importantly, the mouse footpad technique has now been added to the list of assessments in pilot trials. This technique is used to determine the rate at which Myco. leprae are killed. This is done by harvesting Myco. leprae from biopsies of skin at the beginning, during and end of the trial, and inoculating them into mice. This technique is a more accurate and sensitive method for determining the viability of Myco. leprae than is the MI. By the standard mouse footpad technique the skin of patients under standard dapsone therapy are cleared of viable (infectious) Myco. leprae within 3-4 months. By the same criteria rifampicin therapy clears bacilli from the skin within 3 weeks (Rees et al., 1970). The rapid killing of $M y c o$. leprae by rifampicin is consistent with it being a bactericidal drug, as compared with the bacteriostatic activity of dapsone. Moreover, although with rifampicin the MI falls more rapidly than with dapsone, the fall in the MI with rifampicin lags behind loss of viability of Myco. leprae as determined in the mouse. Thus the mouse technique is superior to the MI with a rapidly killing and bactericidal drug, such as rifampicin. The reason for the apparent discrepancy between these two tests is that the changes in the morphology of an organism take 7-10 days to become manifest.

\section{Long-term Trials}

In principle, the objectives of long-term trials are a logical sequence in determining the final efficacy of a drug or drug combination, using various regimens, to achieve cure. While for tuberculosis and other infectious diseases such long-term trials have a logical basis, because the infections can be cured, this has not been uniformly achieved in lepromatous leprosy using standard dapsone therapy. Therefore, as the primary objective of any chemotherapy has not been achieved in lepromatous leprosy, the primary objective must be to investigate new drugs in the hope of achieving cure. However, the special features of lepromatous leprosy, which have already been discussed in the earlier part of this paper, together with the failure of dapsone and its use only as monotherapy, have in themselves influenced the planning and objectives of long-term trials. Because of these special circumstances I will first pinpoint the problems as revealed from present knowledge using dapsone as monotherapy and the pattern of drug resistance, and recent results using rifampicin. In analysing the present situation I 
shall heavily draw on pharmacological and bacteriological data obtained by using the mouse footpad infection.

I must first recapitulate that the chemotherapy of leprosy had been entirely based on monotherapy and nearly all the 30 years experience has been with dapsone or closely related derivatives. In lepromatous leprosy, for any semblance of cure, dapsone has had to be administered continuously for 5 years at least, frequently 10 years and now it is advised for life. Such prolonged therapy is unique, is impracticable since under no medical services and patient collaboration can such prolonged therapy be maintained. In the last 10 years, using the mouse footpad infection, it has been demonstrated that dapsone resistance can occur or that in spite of apparent continuous dapsone therapy for 10 years, a high proportion of such patients can be shown to harbour a few dapsone sensitive bacilli (Waters et al., 1974). Regarding the emergence of dapsone resistance, the mouse footpad test has shown that by and large dapsone resistant infections take at least 6 years to evolve and can still evolve after 24 years dapsone therapy. The same studies have shown that dapsone is bacteriostatic. While from experience with the chemotherapy of tuberculosis, monotherapy would have been expected to have resulted in drug resistance similarly in leprosy, the prolonged time lag in leprosy is unique.

Two thiourea derivatives-thiacetazone and thiambutosine-have also been used to a limitad extent in the therapy of leprosy, as monotherapy, and with both these drugs clinical relapses have been frequent after 2-3 years. Moreover, recently it has been established that these relapses are due to the emergence of resistant strains, as demonstrated in the mouse footpad infection. Therefore, the emergence of drug resistance to monotherapy by the thioureas has occurred much more rapidly than with dapsone, and within a period that is more consistent with the rate of emergence of drug resistance in tuberculosis.

Another antileprosy drug, clofazimine, has also been used as monotherapy for some 10 years, and to date there is no evidence of drug resistance.

Dapsone, the thioureas and clofazimine have all been shown in the mouse footpad infection to be bacteriostatic drugs.

Still more recently rifampicin has been used for about 5 years in the chemotherapy of leprosy, again largely as monotherapy, and to date no drug resistance has been reported. Rifampicin, on the other hand, differs from all the other antileprosy drugs in being bactericidal. However, as reported by us elsewhere in this Colloquium, where we have monitored in mice homogenates of skin and other tissues from patients treated continuously with rifampicin for up to 2 years, a significant proportion of such patients have been shown to still harbour some living Myco. leprae.

On the basis of our experience with dapsone and all the data on other antileprosy drugs, we have now to decide the purpose for which future long-term trials should be undertaken and the general design and feasibility of such trials that are likely to improve the therapy of lepromatous leprosy. The primary reason for a long-term trial of a new drug that has proved efficacious in a pilot trial is to establish its continuing efficacy in controlling the infection as judged by clinical and bacteriological assessments. For leprosy, this would be a controlled trial comparing a group of patients on the new drug with a group on dapsone and the bacteriological assessment would be based on the rate of fall in the BI and the time taken for the BI to reach 0 . This simple but logical approach will certainly identify new drugs less efficacious than dapsone, and identify new drugs giving 
rise to drug resistance within 2-3 years, like thiambutosine and thiacetazone. On this basis, a long-term trial of 5 years would suffice. However, as we now know dapsone therapy alone is not necessarily a cure when maintained for 10 years or more because even when the BI is 0, dapsone sensitive viable organisms may persist and the patient relapse when treatment is stopped. Furthermore, dapsone treated patients can relapse with resistant organisms at any time after 6 years or more of continued dapsone therapy. In order to exclude this pattern of resistance for a new antileprosy drug, controlled long-term trials would have to be continued, with large groups of patients, for at least 10 years. It is on the basis of these two phenomena associated with dapsone therapy, and the fact that dapsone and all other antileprosy drugs have been administered as monotherapy, we need to reappraise the objectives for long-term trials. From the experience of chemotherapy in tuberculosis there is overwhelming evidence that all antituberculosis drugs given as monotherapy universally result in drug resistance. Because monotherapy has so far been universally applied in leprosy and because dapsone is standard therapy, the most important long-term controlled trials which need to be undertaken in leprosy must be designed to establish whether a second drug given with dapsone significantly reduces the incidence of dapsone resistance. However, such a controlled trial would require at least 200 previously untreated lepromatous patients and would have to be continued for not less than 10 years. Unless a trial of this type is undertaken the role of combined therapy in leprosy will remain unanswered. Because of the danger of drug resistance there is a case for assuming without proof the efficacy of combined therapy because of the difficulties and delay in undertaking a trial. However, even if this principle was accepted, long-term trials would still be required to assess all new drugs which would then always have to be given in combination with, for example, dapsone or another known antileprosy drug. While resistance is one major reason for undertaking long-term trials, there is still the persister problem revealed from experience with dapsone, which also can only be investigated by long-term studies. Persisting viable bacilli and the need for prolonged therapy may well be associated and due to dapsone being a bacteriostatic drug. Therefore the discovery of rifampicin as the first bactericidal drug against Myco. leprae can now be used to test this hypothesis, and a number of trials are under way. However, preliminary results show that some viable bacilli persist still in patients treated daily with rifampicin for 2 years. Therefore rifampicin has not reduced the treatment time dramatically although it may well shorten the 10 years or more required for dapsone. Nevertheless viable organisms after 2 years treatment with a powerful bactericidal drug may indicate that chemotherapy alone will not cure lepromatous patients because of their diminished immunological competence. Further long-term trials alone will answer this important question. If the results then show that rifampicin is no more effective than dapsone, trials with combined immunotherapy will have to be investigated.

\section{References}

Committee Report on Advances in Experimental Chemotherapy (1974). 10th Internat. Leprosy Congr. 1973. Lepr. Rev. 45, 48.

Doull, J. A. (1960). Controlled clinical trials in leprosy. W.H.O. Geneva. Document $\mathrm{MHO} / \mathrm{PA} / 97.60$.

Editorial (1974). Tuberculosis of the spine. Lancet, i, 137.

Rees, R. J. W., Pearson, J. M. H. and Waters, M. F. R. (1970). Experimental and clinical studies on rifampicin in the treatment of leprosy. Br. med. J. 1,89. 
Ridley, D. S. and Jopling, W. H.(1966). Classification of leprosy according to immunity. A five-group system. Int. J. Lepr. 34, 255.

Ridley, D. S. and Waters, M. F. R. (1969). Significance of variations within the lepromatous group. Lepr. Rev. 40, 143.

Waters, M. F. R., Rees, R. J. W., McDougall, A.C. and Weddell, A. G. M. (1974). Ten years of dapsone in lepromatous leprosy: Clinical, bacteriological and histological assessment and the finding of viable leprosy bacilli. Lepr. Rev. 45, 288.

Waters, M. F. R., Rees, R. J. W. and Sutherland, I. (1967). Chemotherapeutic trials in leprosy. 5. A study of methods used in controlled chemotherapeutic trials in lepromatous leprosy. Int. J. Lepr. 35, 311. 